2

7

\author{
A. Akkaoui*, S. Caré, M. Vandamme \\ Laboratoire Navier, UMR 8205, École des Ponts, IFSTTAR, CNRS, UPE, \\ Champs-sur-Marne, France
}

\begin{abstract}
6 Abstract
${ }_{8}$ The objective is to determine the relevant microstructural and mechanical

9 parameters of the constituents that influence its elastic properties. To reach

In this work we aim to study the elastic behavior of wood-aggregate concrete. this objective, we combined experiments and modeling. Experimental results show that the evolution over time of the elastic properties of wood-aggregate concrete is complex: it depends on storage conditions, content of binder and drying time. To well understand this evolution, we developed a micromechanical model based on the self-consistent scheme. This model captures well the experimental data and provides a predictive tool for the behavior of plant-based concrete.

Keywords: concrete, wood-aggregate, elastic moduli, Digital Image

Correlation, modeling, self-consistent scheme
\end{abstract}

\section{Introduction}

Light-weight concrete composed of particles of plant origin and a mineral binder is increasingly studied. This concrete appears to be an environmentally-

\title{
Experimental and micromechanical analysis of the elastic properties of wood-aggregate concrete
}

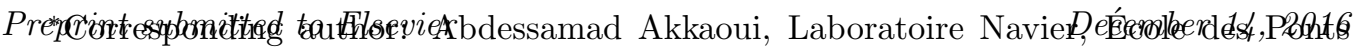
ParisTech, 6 et 8 avenue Blaise Pascal 77455 Marne-la-Vallée cedex 2 FRANCE; Email, abdessamad.akkaoui@enpc.fr 
friendly material $[1,2]$. Thermal performance of concrete is enhanced by the use of plant-based particles like hemp, wood, or flax shives [3-5]. Moreover, plant-based concrete is characterized by sound insulation owing to its permeability and its high open porosity [6]. Using this building material in construction makes it possible to reduce greenhouse gas emissions [1] but also to value by-products from other industries (e.g., woodwork activities or agriculture). This material may be subjecyed to mechanical loadings. For this reason, a better knowledge of its mechanical properties is required.

When studying experimentally the mechanical behavior of plant-based concrete, the following properties are usually measured: compressive strength, flexural strength and elastic properties. The stress/strain curve obtained from compression or flexural test on this material is however rarely described in the literature. Cérézo [6] observed that, for hemp concrete, the curve obtained from compression testing generally presents a peak stress, before which the load is carried by the binder, and after which the load is carried by the aggregates. According to Nguyen et al. [7], the mechanical behavior is, at the beginning of the test, elastic, with a homogeneous strain field over the entire sample. Then the binder matrix is deteriorated and the stresses are redirected toward the hemp particles that harden until eventual failure of the specimen. These results need to be extended to other types of plant-based particles.

The mechanical behavior of plant-based concrete depends on its microstructure. Several authors [8-11] reported that increasing the content of 
binder in plant-based concrete leads to higher mechanical properties. Compacting the concrete during its manufacturing is also a parameter that increases its mechanical properties, as shown by Cérézo [6] and Nguyen et al. $[12]$.

To improve the understanding of the mechanical behavior of this concrete, homogenization models were reported in the literature. These models can be classified into two categories: analytical $[6,13]$ and numerical models $[13,14]$. Analytical models use spherical (bicomposite or tricomposite) or ellipsoidal inclusions and are based on the classical homogenization schemes (e.g., self-consistent, Mori-Tanaka, and Hashin and Shtrikman). Numerical modeling aimed principally to predict mechanical behavior of morphologically rich concretes (e.g., multi-phasic materials with bar shaped particles and high porosity rates [14]) in the framework of linear and nonlinear elasticity by iterative homogenization schemes. The developed model used plate shape inclusions and finite element calculations. For these analytical and numerical models, the simulated results are satisfactory $[6,13,14]$.

However, in these models, two limitations can be mentioned. The first one comes from the fact that the elastic properties used for the binder were often measured on a free piece of this material, while the presence of the plant-based particles can modify the behavior of the binder: i) the possible exchange of water between the particles and the binder makes it difficult to estimate the actual value of the water-to-cement mass ratio $w / c$; ii) the presence of plant-based particles influences the hydration process and then the 
mechanical properties of the binder; iii) the porous characteristic of plantbased concrete induces an emphasized coupling of hydration and drying in the material leading to a possible damage of the binder. The second limitation is related to the inverse analysis to overcome the difficulties of determining experimentally the elastic properties of the plant-based particles. These difficulties come from: i) the possible effect of different treatments often applied to the aggregates on their mechanical properties; ii) the variability of the mechanical properties within the same species of plant-based particles; iii) the evolution of these properties with the water content of the particles [15]; and iv) for some plant-based particles (e.g., hemp and flax shives), the difficulty to measure these properties in their directions of anisotropy. For all these reasons, the inverse analysis is often used to back-calculate the elastic properties of the plant-based particles. It is however necessary to discuss the validity of these back-calculated properties.

The work reported here focuses especially on the study of elastic properties of wood-aggregate concrete using both experimental and theoretical approaches. It aims at investigating a "model" material to determine the effect of the properties of each constituent on the global behavior of the concrete. Only non-compacted concrete is studied in our work. We first measured the Young's modulus of the material at different ages using digital image correlation. Then, we developed a homogenization model based on the self-consistent scheme, that we validate and discuss based on the experimental results. 


\section{Experimental study of the elastic properties of wood-aggregate} concrete

\subsection{Materials and methods}

We studied mixtures of wood-aggregate concrete with identical content of wood aggregates per unit volume and water-to-cement mass ratio $(w / c=0.5)$ but with various proportions of cement paste. The cement used was a CPACEM II/B-LL 32.5 supplied by Lafarge (Saint-Cloud, France). The mineral composition of this cement determined by X-ray diffraction, performed at École des Mines de Douai, is given in Table 1. This cement is characterized by a density of 0.299 , a Blaine surface area of $0.406 \mathrm{~m}^{2} \cdot \mathrm{g}^{-1}$ and a compressive strength at 28 days of $40.8 \mathrm{MPa}$. The wood shavings were supplied by Agresta Technologies (Pouxeux, France) and were obtained from spruce. Their commercial name is AGRESLITH-C and their characteristic size ranges from 4 to $10 \mathrm{~mm}$. Those shavings underwent both thermal and physico-chemical treatments to limit their absorption of water and then enhance their dimensional stability. Their water content (defined as the water-to-dry-wood mass ratio) was measured by drying a sample in an oven at $103^{\circ} \mathrm{C}$ for 48 hours and was equal to $68.7 \%$. Their bulk density $\rho_{b}$, defined as the mass of aggregates divided by the total volume of the bunch they constitute, as provided by Agresta, is 0.22-0.23 g.cm ${ }^{-3}$, while the apparent density $\rho$ of wood, defined as its mass per unit volume, is $0.69-0.70$ g. $\mathrm{cm}^{-3}$.

In general, plant-based concrete is a cavernous material since it presents a high volume of voids (porosity). This porosity is generally classified into 
Table 1: Mineral composition of the cement used, obtained by X-ray diffraction.

\begin{tabular}{|l|c|c|c|c|c|c|c|}
\hline Mineral & $\mathrm{C}_{3} \mathrm{~S}$ & $\mathrm{C}_{2} \mathrm{~S}$ beta & $\mathrm{C}_{3} \mathrm{~A}$ cubic & $\mathrm{C}_{4} \mathrm{AF}$ & Calcite & Gypsum & Quartz \\
\hline Mass fraction (\%) & 56.67 & 2.44 & 2.55 & 4.94 & 29.07 & 3.53 & 0.80 \\
\hline
\end{tabular}

two categories: the intra-phase porosity (where the phase is the aggregate or the binder), which takes into account all voids in the binder and in the plant-based particles, and the macroscopic porosity (also called intergranular porosity) representing the voids between the skeleton. This intergranular porosity is created by the imperfect arrangement of particles when the amount of binder is insufficient to fill completely these voids. In this work, the quantity of wood aggregates, considered as a granular medium, was such that shavings were at their maximum random close packing (i.e., the ratio of their bulk density $\rho_{b}$ to the density $\rho$ of wood was considered equal to $33 \%$ $[2])$, while the content of cement was added in such amounts that the concrete kept a cavernous structure. We chose four cement-to-wood mass ratios $c / a$ : $c / a=1.25,1.75,2.25$, and 2.75 . These proportions are not the ones used in commercialized concretes, they were chosen to evaluate the influence of the quantity of binder on the mechanical properties. Figure 1 shows pictures of the four mixtures.

As the cement paste fills the inter-particle porosity, the volume fractions of the two phases (i.e., wood aggregates and cement paste) are defined by the ratio of the volume of the phase to the apparent volume of wood aggregates required to prepare the samples. Assuming that the cement paste fills the inter-particle porosity without modifying the apparent volume (since the 
layer of cement paste coating the aggregates is only a fraction of their diameter, as seen in the X-ray microtomography image presented in Figure 2 of a sample of wood-aggregate concrete studied in [2]), the latter was considered as the total volume of the samples. Table 2 summarizes the quantities of used materials and the resulting volume fractions. It should be noted that the volume fractions of cement paste presented in Table 2 were calculated assuming that the aggregates do not exchange water with the cement paste during the mixing process. We assume that this exchange of water can be neglected in our case given the treatments that the aggregates underwent and the used mixing process (i.e., we first mixed water and cement and then we added gradually the aggregates). In the case of a potential exchange of water during mixing, the volume fraction of cement paste should be modified accordingly since it is the sum of the volume of the cement powder and of the water available for hydration.

Table 2: Quantities of materials used and calculated volume fractions of studied mixtures. $c / a$ is the cement-to-wood mass ratio.

\begin{tabular}{|l|l|c|c|c|c|}
\hline & $c / a$ & 1.25 & 1.75 & 2.25 & 2.75 \\
\hline \multirow{3}{*}{ Mass of materials (g) } & aggregate & 1407.1 & 1407.1 & 1407.1 & 1407.1 \\
& cement & 1758.9 & 2462.5 & 3166.1 & 3869.6 \\
& water & 879.5 & 1231.2 & 1583.0 & 1934.8 \\
\hline \multirow{3}{*}{ Volume fractions (\%) } & aggregate & 33 & 33 & 33 & 33 \\
& cement paste & 22.3 & 31.2 & 40.1 & 49.0 \\
& air & 44.7 & 35.8 & 26.9 & 18.0 \\
\hline
\end{tabular}

We used a standard mixing machine to mix the constituents of concrete. We first mixed cement and water. Then, we gradually introduced the wood 

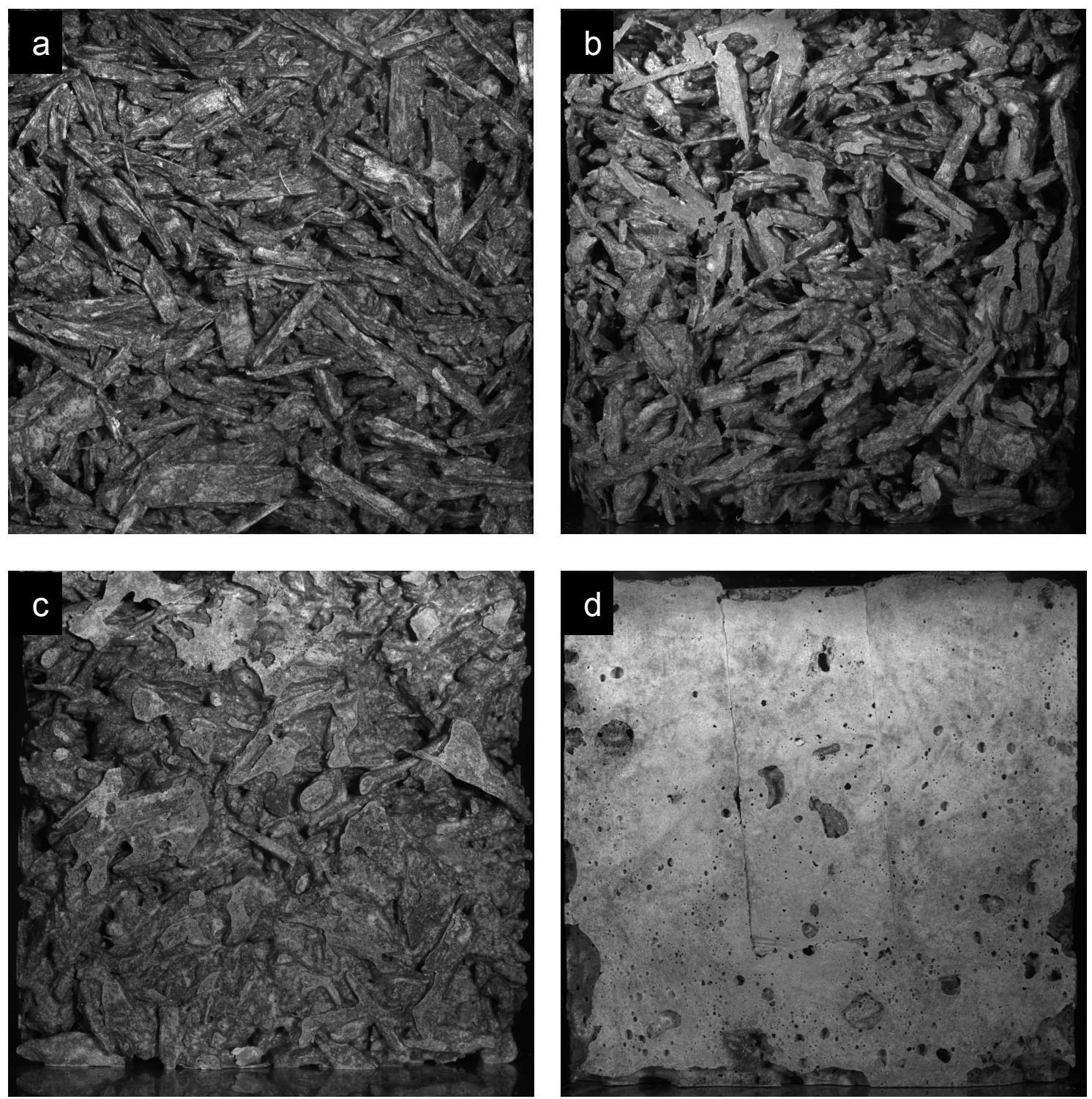

Figure 1: Pictures of the studied wood-aggregate concrete with various cement-to-wood mass ratios: a) $c / a=1.25$, b) $c / a=1.75$, c) $c / a=2.25$, and d) $c / a=2.75$.

aggregates. The total mixing time was 7 minutes. For each mixture, we pre-

151 pared several cubic specimens with dimensions of about $7 \times 7 \times 7 \mathrm{~cm}^{3}$. These

152 dimensions are sufficient taking into account the size of the aggregates (which 


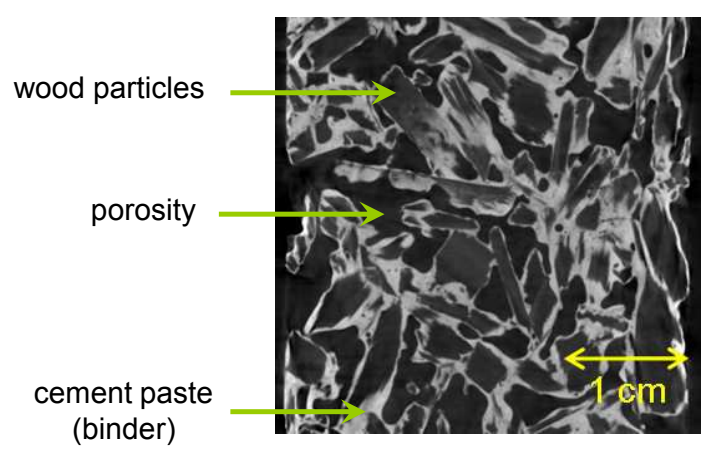

Figure 2: X-ray microtomography image of a sample of wood-aggregate concrete with $c / a=2$ studied in [2].

ranges from 4 to $10 \mathrm{~mm}$ ). The samples were kept in sealed conditions and demolded two days after casting. Then they were subjected to various stages of storage conditions. Two series of samples were subjected to two different conditions: a first series was kept in sealed conditions (by wrapping the samples into plastic films) until the day of testing; while a second series of samples was in humid conditions (i.e., relative humidity $h_{r} \geq 90 \%$, temperature $\left.T=20^{\circ} \mathrm{C}\right)$ for 8 days and then at room conditions $\left(h_{r} \simeq 60 \%\right.$, $T=20^{\circ} \mathrm{C}$ ), which is closer to the conditions of use of the material.

We also prepared cubic samples, with dimensions of about $7 \times 7 \times 7 \mathrm{~cm}^{3}$, of cement paste with a water-to-cement mass ratio $w / c=0.5$ and we kept them, as for the second series of samples, in humid conditions (i.e., relative humidity $h_{r} \geq 90 \%$, temperature $T=20^{\circ} \mathrm{C}$ ) for 8 days and then under room conditions $\left(h_{r} \simeq 60 \%, T=20^{\circ} \mathrm{C}\right)$ until the day of testing.

We used a MTS compression machine with a maximum capacity of 100 $\mathrm{kN}$ to perform uniaxial compression tests on our cubic samples, at different ages up to 104 days. For each test, four loading/unloading cycles with an 
increasing maximum load were performed followed by a last loading up to failure. The loading rate was $10 \mathrm{~mm} / \mathrm{min}$. The compressing machine was equipped with a force sensor to assess the stress applied to the material. For the strains, because of the difficulty in using strain gauges or displacement sensors due to the porous nature of the material, they were determined using $2 \mathrm{D}$ digital image correlation. The principle of this technique is to acquire during the test images of the tested sample, i.e., to follow a face of the specimen while subjected to compression. Figure 3 presents the experimental device used in this study. This device is composed of: the press, a lighting system and a mounting base on which is fixed an acquisition camera. This camera is a Pike F-421B/C with a resolution of $2048 \times 2048$ pixels. The image acquisition rate was set at $2 \mathrm{~Hz}$.

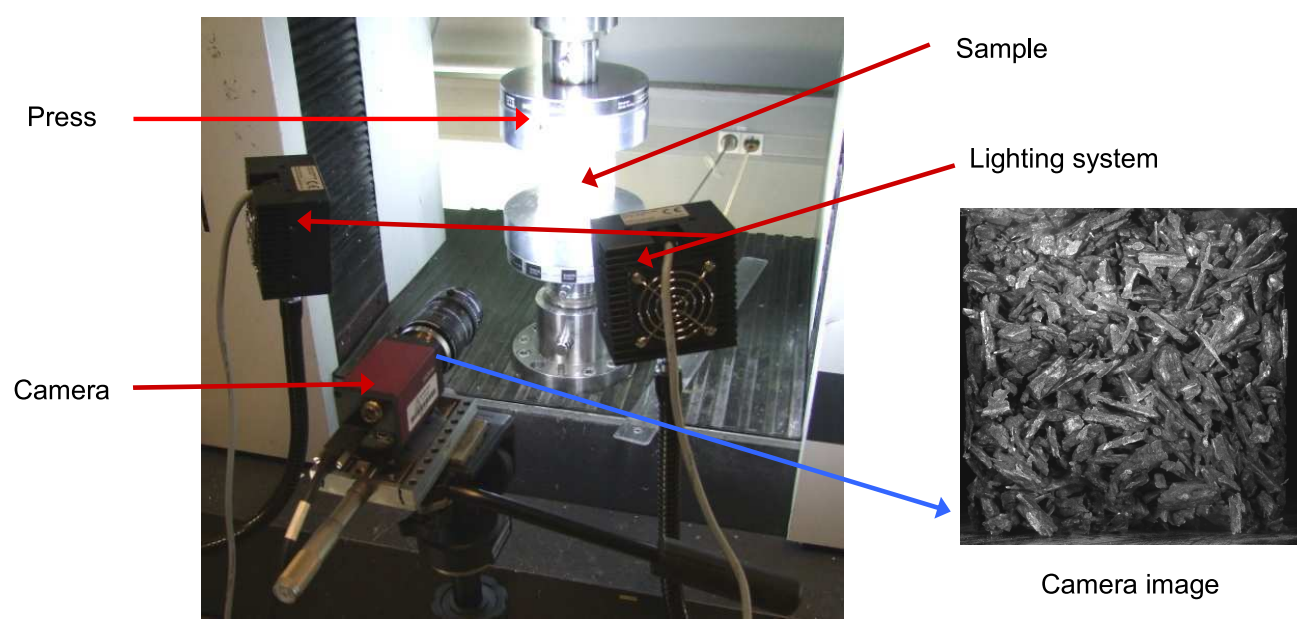

Figure 3: Experimental device used to perform compression tests.

The digital image correlation (DIC) method [16] provides access to the 2D displacement field and hence to the strains of the sample during the 
test. In this study, we used the in-house CMV code [17] for processing the acquired images. The size of the correlation window was $40 \times 40$ pixels. The combination of the strains provided by this code with the stresses determined with the compression machine allowed us to study the mechanical behavior of the tested material. Only one test was performed at each age, but the repeatability was checked on three samples with $c / a=1.25$ at 42 days: the coefficient of variation (i.e., the standard deviation divided by the absolute value of the arithmetic mean) of the measured Young's modulus was $10 \%$.

\subsection{Young's moduli of cement paste and spruce wood}

On the samples of cement paste described in section 2.1, we performed compression tests at 21 and 28 days. The resulting Young's moduli are $6695 \pm 86 \mathrm{MPa}$ at 21 days and $9536 \pm 543 \mathrm{MPa}$ at 28 days. Le Roy [18] proposed the following formula to estimate the Young's modulus $E_{p}$ of cement paste at 28 days:

$$
E_{p}=k_{p e} \frac{K R_{c}}{\left(1+3.15 \frac{w / c}{(1+0.15(1-\exp (-11 s / c)))}\right)^{2.4}}
$$

where $k_{p e}=220, R_{c}$ is the standard compressive strength (i.e., strength measured on cement mortars by the test procedures in the European standard EN 197) of cement paste at 28 days $\left(R_{c}=41 \mathrm{MPa}\right.$ for the CEM II type cement used in this work according to the specifications provided by the manufacturer), $w / c$ is the water-to-cement mass ratio (0.5 in our case), $s / c$ is 
the silica fume-to-cement mass ratio (0 in our case) and $K$ is a proportionality coefficient ( $K=8$ for the experiments of Marchand et al. quoted by [18]). Using this formula, the Young's modulus estimated for our cement paste was $7455 \mathrm{MPa}$. Taking into account the uncertainties of the parameters of the proposed formula, the value we measured for our cement paste, even if it is slightly higher than the estimated one, remains in the right order of magnitude.

For spruce wood, which is an anisotropic material, we performed compression tests in the three main directions of this material: the longitudinal direction (that of the fibers), the radial direction (the direction of growth in diameter), and the tangential direction. The tests were carried out on pieces of solid wood that had not undergone any treatment. Pieces were stored in an ambient environment such that their water content (defined as the water-to-dry-wood mass ratio) was around $7 \%$. The size of the samples was about $30 \times 30 \times 100 \mathrm{~mm}^{3}$ for longitudinal tests, and about $30 \times 30 \times 30 \mathrm{~mm}^{3}$ for radial and tangential tests. The measured Young's moduli were 13700 $\mathrm{MPa}, 1090 \mathrm{MPa}$ and $800 \mathrm{MPa}$ in the longitudinal, radial and tangential directions, respectively. These measurements are slightly higher compared to bibliographic data summarized in Table 3, which were obtained at water content of $12 \%$. Note that Sitka and Engelmann in this table are two types of spruce wood. However, according to Gerhards [15], the Young's moduli of wood depend on its water content. We can estimate the stiffness that the spruce we tested should exhibit at water content of $12 \%$ using the following 
corrections [19]:

$$
\begin{aligned}
& E_{l}^{h}=E_{l}^{12}(1-0.015(h-12)) \\
& E_{v}^{h}=E_{v}^{12}(1-0.030(h-12)),
\end{aligned}
$$

Table 3: Elastic properties of spruce wood in its three principal directions: longitudinal
$E_{l}$, radial $E_{r}$, and tangential $E_{t}$ Young's modulus; and Poisson's ratios $\nu_{r l}, \nu_{t r}$ and $\nu_{l t}$ at

Table 3: Elastic properties of spruce wood in its three principal directions: longitudinal
$E_{l}$, radial $E_{r}$, and tangential $E_{t}$ Young's modulus; and Poisson's ratios $\nu_{r l}, \nu_{t r}$ and $\nu_{l t}$ at water content of $12 \%$.

\begin{tabular}{|l|c|c|c|c|c|c|}
\hline Reference & $E_{l}(\mathrm{MPa})$ & $E_{r}(\mathrm{MPa})$ & $E_{t}(\mathrm{MPa})$ & $\nu_{r l}$ & $\nu_{t r}$ & $\nu_{l t}$ \\
\hline$[20]$ & 10700 & 649 & 348 & 0.02 & 0.3 & 0.4 \\
{$[21]$ Sitka } & 10890 & 849 & 468 & 0.04 & 0.248 & 0.467 \\
[21] Engelmann & 9790 & 1253 & 578 & 0.083 & 0.255 & 0.462 \\
Our data & 12760 & 950 & 700 & - & - & - \\
\hline
\end{tabular}

where $E_{l}^{h}$ and $E_{l}^{12}$ are the longitudinal Young's moduli at a water content of $h$ and 12 (in percent), respectively; $E_{v}^{h}$ and $E_{v}^{12}$ are the Young's moduli in the direction $v(v=r$ for radial and $v=t$ for tangential) at a water content of $h$ and 12 (in percent), respectively. The resulting values are presented in Table 3. These values of Young's moduli extrapolated to a water content of $12 \%$ are consistent with literature data $[20,21]$ obtained at the same water content.

\subsection{Mechanical behavior of wood-aggregate concrete}

\subsubsection{Stress/strain curves}

Figure 4 shows typical stress/strain curves obtained by compression of wood-aggregate concrete. The analysis of these curves shows that the mechanical behavior of this material is complex and depends on its content of 

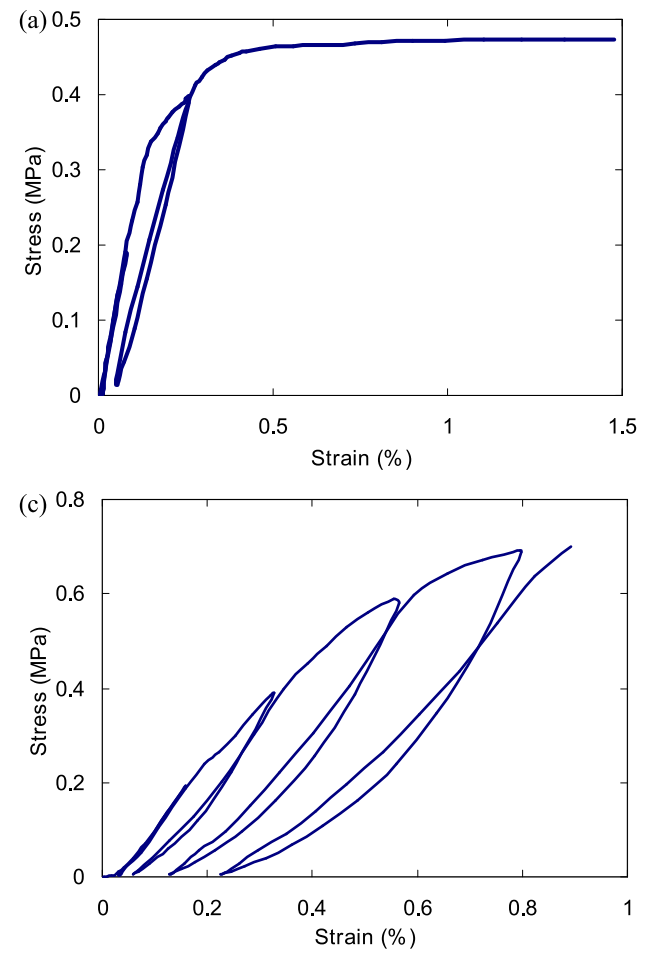
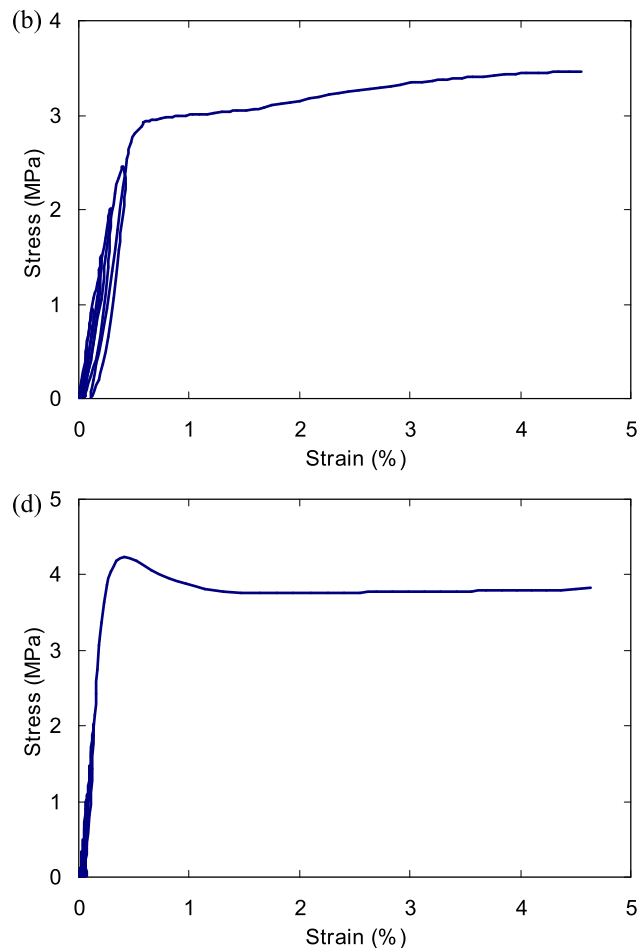

Figure 4: Typical behavior of wood-aggregate concrete: (a) with a low content of cement, (b) with an intermediate content of cement, (c) zoom in cyclic loading, and (d) with a high content of cement.

After the elastic phase of the behavior, residual strains appear, as we observe in Figure 4(c). These residual strains, which indicate an elastoplastic 
behavior, might be due to the damage of the binder (i.e., progressive cracks in the cement matrix and/or in the aggregate/binder interfaces). This phase ends by a possible failure of the binder.

After the failure of the binder, we observed two main types of behavior depending on the content of binder. In the case where this content of binder is high, we observed a peak in the stress/strain curve (see, e.g., Figure $4(\mathrm{~d}))$. This peak could be due to the fact that (1) the behavior of the binder is brittle and (2) the stiffness of the aggregates is lower than that of the binder [6], thus explaining the decrease in the stress supported by the material. The peak is followed by a plateau corresponding to the compaction of the granular skeleton, making the material denser (closing intergranular and possibly intragranular porosities). In contrast, stress/strain curves of wood-aggregate concrete with low content of binder show no peak (see, e.g., Figure 4(a)). The plateau corresponding to the compaction of the aggregates is usually the maximum stress. The absence of a peak may be explained by the fact that, at low content of binder, only a thin layer of cement paste is surrounding the aggregate, so that the fraction of the loading supported by the aggregates is significant.

These results are, in general, consistent with those of the hemp concrete studies $[6,7,12]$. It therefore seems that this behavior characterizes the concrete made of plant-based aggregates.

We used the stress/strain curves of the compression tests to determine the Young's modulus of the studied concrete. This Young's modulus is calculated 
from the linear portion of the curves. As we performed load/unload cycles on each tested specimen, the Young's modulus is the average of the moduli on these cycles, as illustrated in Figure 5.

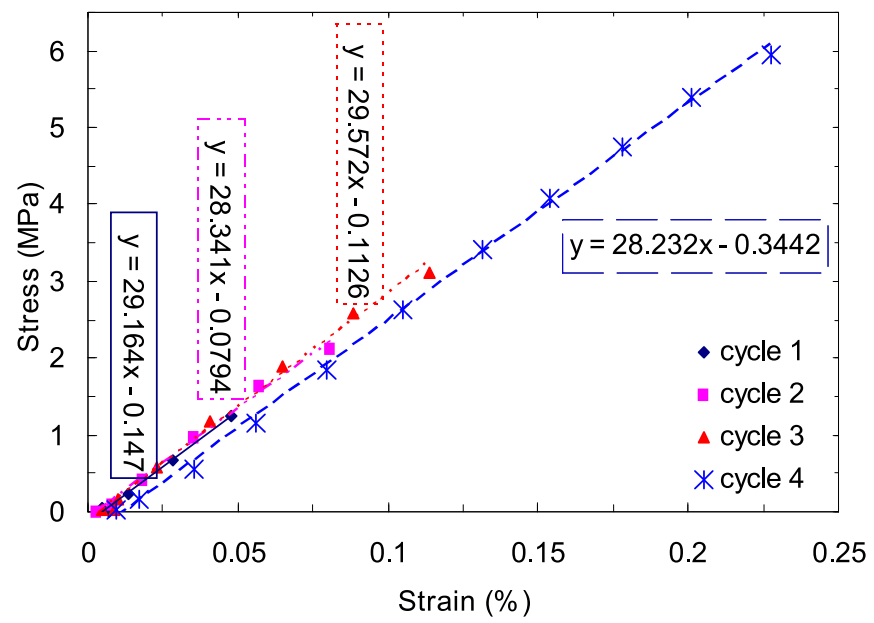

Figure 5: Typical case of determining the Young's modulus of wood-aggregate concrete from the stress/strain curve.

\subsubsection{Young's modulus}

We performed compression tests on the first series of samples (i.e., the one kept in sealed conditions), as described in section 2.1, at the age of 28, 62 and 104 days. Table 4 presents the mass losses of the tested samples. This table shows that the mass of the samples decreased slightly (less than 4\%) under the studied conditions. The reason can be that the films we used did not completely prevent exchange of humidity between our samples and the external environment. Table 5 summarizes the measured Young's moduli. For all studied mixtures, the Young's modulus increased between 28 and 62 days. This increase might be attributed to the fact that, in absence of 
exchange of humidity with the external environment, the hydration process continues to progress after 28 days. The water consumed by the hydration may be balanced by the transfer of humidity from wood aggregate to cement paste, which makes the hydration continue and enhances accordingly the elastic properties of the concrete. However, between 62 and 104 days, we observed an opposite trend for samples with low content of cement paste (i.e., samples with $c / a=1.25$ and $c / a=1.75$ ). For concrete with $c / a=2.25$, the measured Young's modulus at 104 days was very high (nearly twice) compared to that at 62 days. These two trends after 62 days may be ascribed to the fact that some samples underwent a significant damage while others did not. Note that the Young's modulus of the concrete with $c / a=2.75$ at 104 days could not be measured because of a problem during the test.

Table 4: Mass loss (\%) of samples of wood-aggregate concrete in sealed conditions.

\begin{tabular}{|c|c|c|c|}
\hline Composition $\quad$ Age (days) & 28 & 62 & 104 \\
\hline$c / a=1.25$ & 1.3 & 2.2 & 3.4 \\
\hline$c / a=1.75$ & 0.5 & 2.1 & 3.7 \\
\hline$c / a=2.25$ & 0.8 & 1.6 & 2.6 \\
\hline$c / a=2.75$ & 0.4 & 1.1 & 3.1 \\
\hline
\end{tabular}

Table 5: Young's modulus (MPa) of the wood-aggregate concrete in sealed conditions.

\begin{tabular}{|c|c|c|c|}
\hline $\begin{array}{ll} & \text { Age (days) } \\
\text { Composition } & \end{array}$ & 28 & 62 & 104 \\
\hline$c / a=1.25$ & 170.6 & 228.9 & 150.6 \\
\hline$c / a=1.75$ & 281.8 & 482.4 & 409.7 \\
\hline$c / a=2.25$ & 650.1 & 857.8 & 1680.7 \\
\hline$c / a=2.75$ & 1102.6 & 1643.6 & - \\
\hline
\end{tabular}


As described in section 2.1, the second series of samples was kept in conditions of use (i.e., humid conditions with relative humidity $h_{r} \geq 90 \%$ for 8 days then at room conditions with $h_{r} \simeq 60 \%$ ). Figure 6 a displays the decrease in mass of the samples under these conditions. The difference of storage conditions resulted in two rates of mass loss: lower up to 8 days (mass decreased by less than 3.5\%) and higher after 8 days, as long as humidity balance between samples and the outside environment was not achieved (which occurred at around 30 days). Figure $6 \mathrm{~b}$ presents the evolutions with time of Young's moduli of wood-aggregate concrete until the age of 28 days in conditions of use. With identical wood content, the Young's modulus increased when the content of cement increased. This result is consistent with the fact that the cement paste fills the intergranular porosity. The higher the content of cement, the less porous the wood-aggregate concrete, and the higher its Young's modulus. Figure 6b shows also that the Young's modulus of wood-aggregate concrete increased with its age up to 28 days. This increase in Young's moduli is a result of the evolution of two processes: hydration and drying. The hydration of cement paste may enhance its elastic properties, and drying of wood-aggregate concrete may induce an increase in elastic properties of both cement paste and wood aggregates. It is however difficult to identify the contribution of each of the processes on the raise of Young's modulus of the concrete.

After the age of 28 days, two trends can be distinguished, as seen in Figure 6b: for the concrete with low content of cement paste (i.e., mixtures 


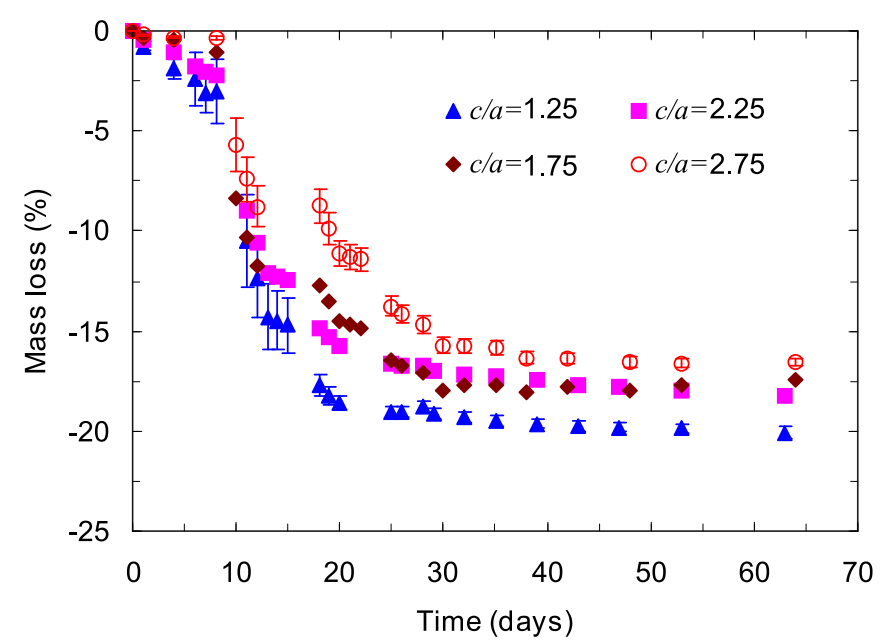

(a)

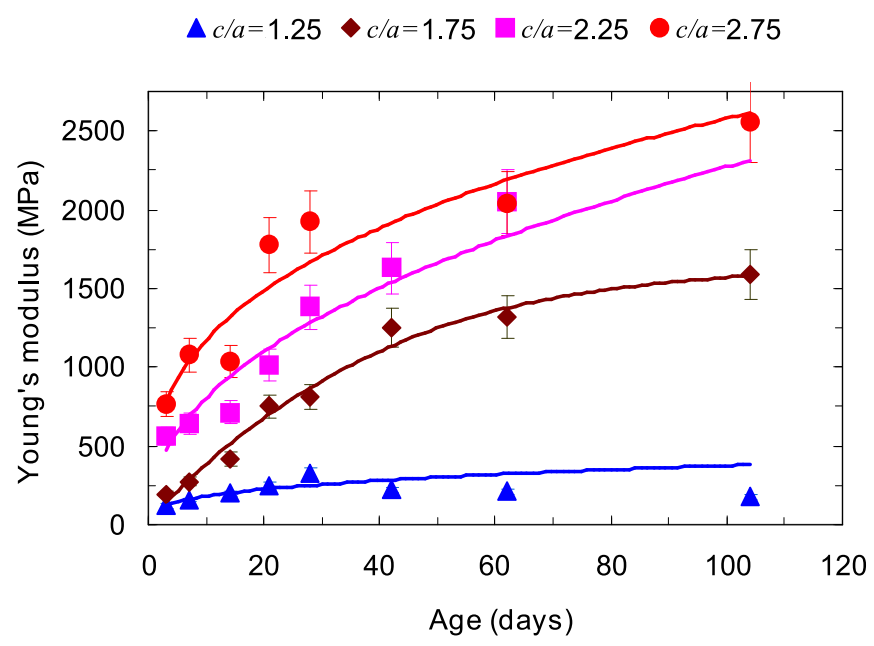

(b)

Figure 6: (a) evolution of the mass loss and (b) Young's modulus of wood-aggregate concrete kept in humid conditions for 8 days then at room conditions.

with $c / a=1.25$ and $c / a=1.75)$, The Young's modulus was stabilized or even decreased (for mixture with $c / a=1.25$ which contains the lowest content of cement paste); while for the concrete with high content of cement 
paste (i.e., mixtures with $c / a=2.25$ and $c / a=2.75$ ), it increased continuously. The mass loss (Figure 6a) indicates that the mass of the samples was stabilized at around 30 days. Drying is then no longer involved in the evolution of the Young's modulus of wood-aggregate concrete after 30 days. The hydration-induced modification of the microstructure of the material or a possible damage of the binder are consequently the main factors driving the change in its Young's modulus in that period. The low content of cement paste in concretes with $c / a=1.25$ and $c / a=1.75$ might then explain their behavior after 28 days, since only a thin layer of binder surrounds the aggregates. Any decline in its quality in time, for example due to damage which seems to be more significant for concrete with low content of cement paste, induces a decrease in Young's modulus of wood-aggregate concrete.

\subsubsection{Partial conclusion}

The experimental results presented above show that the evolution of Young's modulus of wood-aggregate concrete changed its trend after 28 days whatever the storage conditions. This evolution of Young's modulus of wood-aggregate concrete depends in fact on the content of cement paste: mixtures with low content of cement paste $(c / a=1.25$ and $c / a=1.75)$ on the one hand, and those with high content of cement paste $(c / a=2.25$ and $c / a=2.75$ ) on the other hand, have similar behaviors. A possible significant damage of some samples after 28 days (see [22]), especially those with low content of cement paste, may explain this difference. In the next section, 
we present a predictive tool for the elastic behavior of wood-aggregate concrete. Modeling the damage is not the subject of this work, and therefore only results up to 28 days are taken into account.

\section{Micromechanical modeling}

The experimental study presented in the previous section is completed by a modeling work. The purpose of this modeling work is to provide a predictive tool of the elastic behavior of wood-aggregate concrete, in order to enable us to identify relevant parameters influencing this behavior. This predictive tool is based on the technique of micromechanical homogenization. This homogenization aims, in the framework of elasticity, to determine the stiffness tensor of the macroscopic medium, based on the microstructure and the mechanical properties of its different constituents. This exercise generally follows four basic steps [23]: firstly the definition of the representative elementary volume (REV), secondly the choice of an appropriate mechanical loading on this REV, thirdly the determination of localization fields, and finally homogenization per se.

The definition of an REV involves representing an elementary volume of heterogeneous material, by describing its geometry and its constitution, on which the micromechanical analysis will be performed.

The purpose of the localization is to identify the local strain and stress fields resulting from a macroscopically imposed mechanical loading.

The homogenization step consists in determining the stiffness tensor of 
the equivalent medium from the identified mechanical response (strain and stress fields) of the REV to the imposed loading. This determination can be done using only the average of these fields upon each phase (constituent).

We adopt this approach in the next section to derive our micromechanical model.

\subsection{Derivation of the model}

The concrete that we studied is characterized by randomly oriented aggregates, since in the absence of compaction, these aggregates have no preferential orientation. According to the work of Mom et al. [14], taking into account the anisotropy and the shape of the wood aggregate is not necessary to model the elastic properties of concrete incorporating randomly oriented particles. Therefore, we chose spherical shapes to represent wood aggregates in this work.

Figure $7(\mathrm{a})$ schematically shows the microstructure of wood-aggregate concrete based on the observations of section 2.1 (see Figure 1). The microstructure shows that the wood aggregate is surrounded by a layer of binder (with a variable thickness) while air occupies the porosity between these aggregates (intergranular porosity). For this reason, we opted for the microstructural cell shown in Figure 7(b). This microstructural cell consists of two types of inclusions: a 2-layer spherical inclusion representing wood aggregate surrounded by the binder; and a simple spherical inclusion representing intergranular porosity. These inclusions are immersed into the 
homogenized medium whose properties are to be determined.

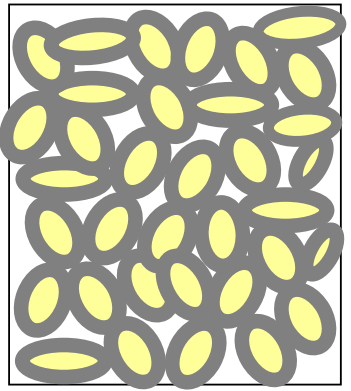

(a)
(1): air

(2): aggregate

(3): binder

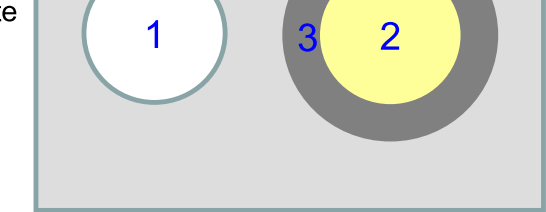

(b)

Figure 7: (a) Simplified microstructure of wood-aggregate concrete and (b) unit cell used to model the elastic properties of that concrete.

Due to the microstructure of the studied material, we chose to use the self-consistent scheme. This scheme is indeed very well suited to the modeling of granular materials for which no phase can be regarded as the matrix [23]. Since the objective is to determine the elastic properties of the studied concrete, we can consider only the linear elastic range of the constituents: the mechanical behavior of cement paste, which is the binder, is isotropic; while that of wood aggregate, being modeled by a spherical inclusion, can also be considered isotropic. Thus, the behavior of the homogenized equivalent medium is also isotropic linear elastic.

To make the approach easier, we transform the problem with two inclusions (i.e., a simple sphere and a 2-layer composite sphere) into two basic problems: the first with one spherical inclusion and the second with one 2layer composite sphere; both are subjected to a uniform strain tensor $\underline{\underline{E}}^{\infty}$ at infinity, as we can see in Figure 8. The problem with a unique spherical 


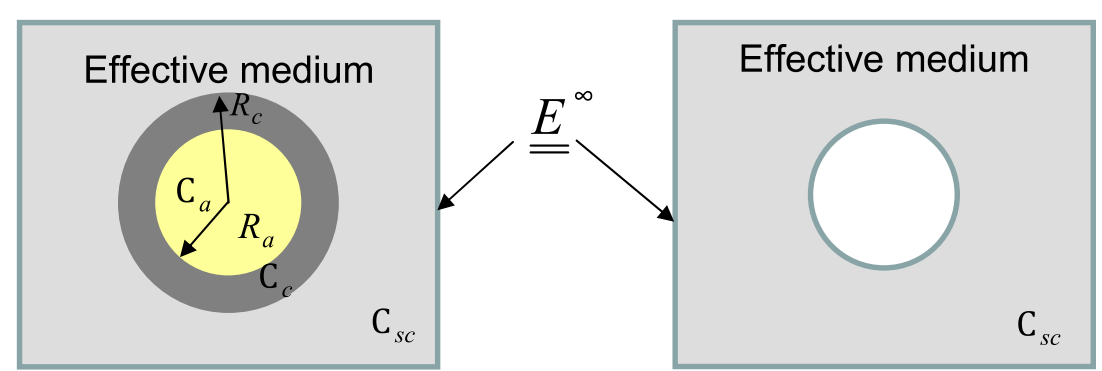

Figure 8: Basic problems with two-layer composites sphere (left) and simple composite sphere (right).

and its the shear modulus $\mu_{h}$ :

$$
\mu_{h}=\frac{f_{a} \frac{1}{\mathcal{S}_{h}}\left(\mathcal{S}_{a}-\frac{21}{5} \frac{R_{a}^{2}}{\left(1-2 \nu_{a}\right)} \mathcal{T}_{a}\right) \mu_{a}+f_{c} \frac{1}{\mathcal{S}_{h}}\left(\mathcal{S}_{c}-\frac{21}{5} \frac{R_{c}^{5}-R_{a}^{5}}{\left(1-2 \nu_{c}\right)\left(R_{c}^{3}-R_{a}^{3}\right)} \mathcal{T}_{c}\right) \mu_{c}}{f_{p} \frac{1}{1-\beta_{h}}+f_{a} \frac{1}{\mathcal{S}_{h}}\left(\mathcal{S}_{a}-\frac{21}{5} \frac{R_{a}^{2}}{\left(1-2 \nu_{a}\right)} \mathcal{T}_{a}\right)+f_{c} \frac{1}{\mathcal{S}_{h}}\left(\mathcal{S}_{c}-\frac{21}{5} \frac{R_{c}^{5}-R_{a}^{5}}{\left(1-2 \nu_{c}\right)\left(R_{c}^{3}-R_{a}^{3}\right)} \mathcal{T}_{c}\right)},
$$


where: $\mathcal{X}_{i}, \mathcal{S}_{i}$ and $\mathcal{T}_{i}$ are constants determined using the boundary conditions (see Appendix A), $R_{i}$ is the radius of the $i$ phase (see Figure 8), $\nu$ is the Poisson's ratio, and $\alpha_{h}$ and $\beta_{h}$ two parameters depending on $k_{h}$ and $\mu_{h}$ [23]. The system of two implicit equations (3) and (4) with two unknowns $k_{h}$ and $\mu_{h}$ can be solved analytically using an algebraic computing tool.

\subsection{Calibration of the model with experimental results}

The system of equations (3) and (4) involves several parameters. The first parameters are the volume fractions. These volume fractions were theoretically calculated and presented in Table 2. The second parameters are the radii $R_{a}$ and $R_{c}$ which appear explicitly in both equations (3) and (4) but also implicitly in the constants $\mathcal{X}_{i}, \mathcal{S}_{i}$ and $\mathcal{T}_{i}$. However, these radii are eliminated since the $R_{a} / R_{c}$ ratio is linked to the volume fractions $f_{a}$ and $f_{c}$ via the following equation:

$$
\frac{\left(R_{a}\right)^{3}}{\left(R_{c}\right)^{3}}=\frac{V_{a}}{V_{c}+V_{a}}=\frac{f_{a}}{f_{c}+f_{a}}
$$

\footnotetext{
The last parameters involved in the system to be solved are the elastic properties of the constituents (i.e., aggregates and cement paste). Bulk and shear moduli are replaced by their expressions in terms of the pair "Young's modulus $E$ and Poisson's ratio $\nu$ ". We consider in hereinafter that the Poisson's ratio of each phase is constant. For the cement paste, the Poisson's ratio is 0.2 , while for wood aggregates (whose behavior was considered isotropic since they are randomly oriented), its value is chosen equal to 0.1 by analogy
} 
with what was done for hemp particles $[6,14]$. This value will be discussed further.

The missing parameters are the Young's moduli of the constituents over time. These moduli are difficult to measure because of the various reasons previously cited in section 1 . For these reasons, we decided to determine these quantities by inverse analysis based on the experimental results presented in section 2.3.2 and check that their orders of magnitude are correct. To do so, we used the following assumptions: (1) the Young's modulus of the cement paste is considered identical for the four $c / a$ ratios at all times; (2) we neglect the evolution over time of the Young's modulus of wood aggregate and we consider it identical for all studied $c / a$ ratios. These assumptions will be discussed later. Moreover, as mentioned in section 2.3.2, we limited the study to the period up to 28 days. We therefore have to calibrate five Young's moduli of cement paste (at 3, 7, 14, 21 and 28 days) and one Young's modulus of wood aggregates, from all measurements. This calibration was performed using a data adjustment function ("NonlinearFit" of Maple Software), which minimizes differences between the model function and the data according to the least squares method.

Figure 9 presents the values of the calibrated Young's moduli for the cement paste and wood aggregates. The values of estimated Young's moduli for the cement paste are generally lower than those we measured on a pure cement paste at 21 and 28 days (see section 2.2). The difference may confirm the modification of the process of hydration of the cement in the 
presence of wood aggregates, a phenomenon observed by Govin et al. [28]. A second explanation for the difference between the measurements and the calibrated values could be the hydration/drying coupling. This coupling is more pronounced for the wood-aggregate concrete than for the bulk cement paste, due to the higher intergranular porosity of the former. The presence of drying can slow down cement hydration when the relative humidity drops below $80 \%$ [29] thus explaining the lower elastic moduli calibrated for the cement paste in our concrete. A possible damage at some interfaces between aggregate and binder would be another reason to justify the low calibrated values: cracks at the interface result in additional porosity and a different transmission of stresses between the aggregate and the cement paste. This damage could be taken into account to enhance the model.

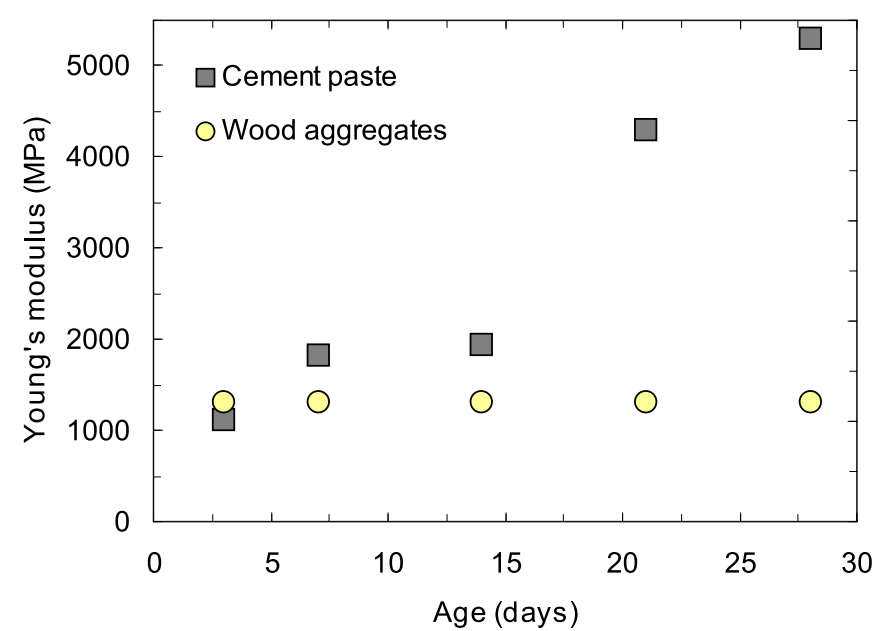

Figure 9: Young's moduli of wood aggregates and of cement paste, calibrated on experimental Young's moduli of wood-aggregate concrete.

${ }_{466}$ The estimated Young's modulus for wood aggregates is compared to an 
equivalent isotropic Young's modulus calculated from the Young moduli and Poisson's ratios of spruce wood in its three anisotropy directions (given in Table 3). Although wood is a heterogeneous material, we can determine its bulk modulus $k$ based on the data of Table 3 (see Appendix B) and, by assuming a Poisson's ratio of 0.1 , deduce an equivalent Young's modulus. Table 6 shows the results. The average value of the Young's moduli obtained from the literature is $1232 \mathrm{MPa}$. This value is consistent with the value which we calibrated, which is $1192 \mathrm{MPa}$. The effect of the variation of the mechanical properties of wood, in particular due to the change in its water content, will be discussed in the next section.

Table 6: Bulk modulus $k$ and estimated equivalent isotropic Young's modulus for the spruce wood.

\begin{tabular}{|l|c|c|}
\hline Reference & Bulk modulus $k(\mathrm{MPa})$ & Equivalent isotropic Young's modulus (MPa) \\
\hline$[20]$ & 377.7 & 906.6 \\
{$[21]$ Sitka } & 662.6 & 1200.3 \\
{$[21]$ Engelmann } & 500.1 & 1590.2 \\
\hline Mean $(\mathrm{MPa})$ & 513.5 & 1232.3 \\
\hline
\end{tabular}

\subsection{Discussion}

In the previous section, we calibrated the model we developed on the experimental measurements. Realistic values were obtained for the calibrated Young's moduli of the constituents of wood-aggregate concrete. We can thus use the model to predict the Young's modulus of the studied concrete, but also to discuss the effect of the properties of the constituents on the elastic behavior of this material. Figure 10 compares the estimated and 


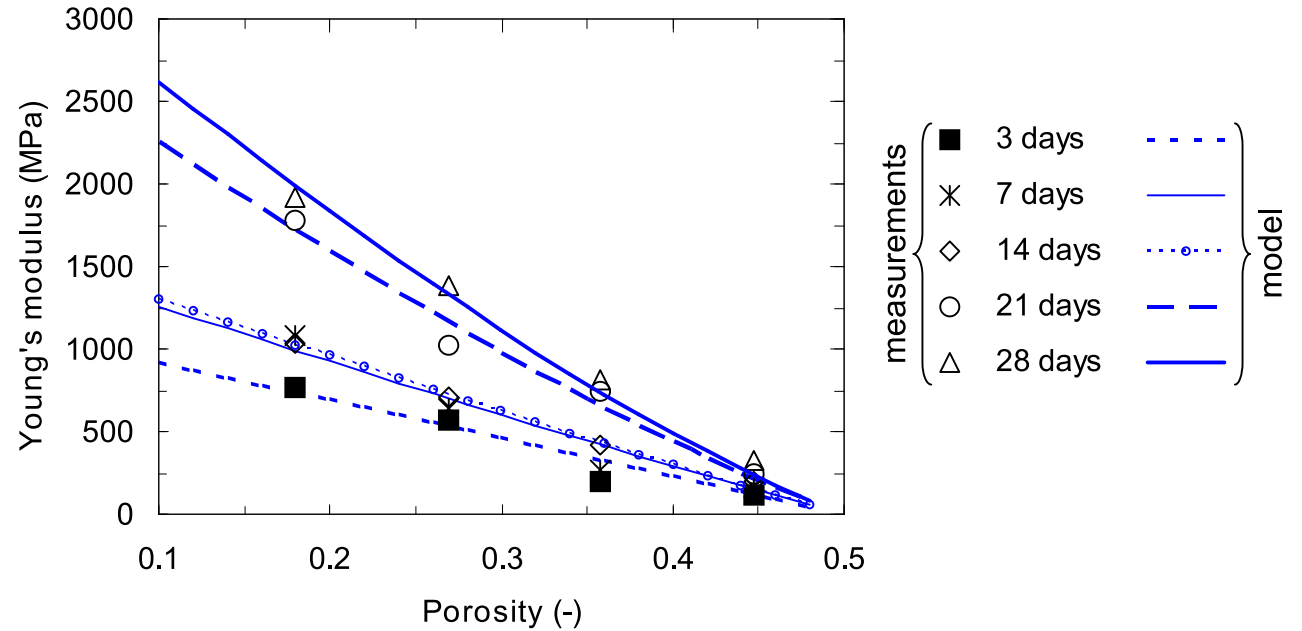
This figure shows that the developed model captures well the experimental measurements over time for all studied $c / a$ mass ratios. The model can then be used as a predictive tool to optimize elastic properties of wood-aggregate concrete.

Figure 10: Young's moduli of wood-aggregate concrete as a function of its intergranular porosity: comparison between the experimental measurements and estimates of the derived model.

measured evolutions of this Young's modulus for different $c / a$ mass ratios.

However, we made some assumptions that should be discussed. The first assumption is the choice of the Poisson's ratio $\nu_{a}$ of wood aggregates. The value of 0.1 was used for modeling elastic properties of hemp concretes [6], [14] but was not discussed. Here, by varying its value, we study the effect of this choice on the Young's modulus estimated with our model. Figure 11a illustrates the results obtained at 28 days for three values of the Poisson's ratio, i.e., $\nu_{a}=0.1,0.2$ and 0.3 . We can clearly see that the three curves are identical, indicating that the Poisson's ratio of the aggregates does not affect 
the Young's modulus of the concrete predicted with our model. However, a comparison of the back-calculated Young's modulus $E_{a}^{\text {calibrated }}$ of the wood with the one $E_{a}^{\text {estimated }}$ derived from bibliographic data (Table 7) shows that the best agreement between the two moduli is obtained for $\nu_{a}=0.1$. For $\nu_{a}=$ 0.2 and $\nu_{a}=0.3$, back-calculation gives values of $E_{a}^{\text {calibrated }}$ equal to $1237 \mathrm{MPa}$ and $1247 \mathrm{MPa}$, respectively; while the values of $E_{a}^{\text {estimated }}$ calculated based on the literature data were $924 \mathrm{MPa}$ and $616 \mathrm{MPa}$, respectively. Therefore, the most appropriate value of the Poisson's ratio for an isotropic representation of the wood particle is $\nu_{a}=0.1$.

Table 7: Calibrated equivalent Young's modulus $E_{a}^{\text {calibrated }}$ of wood aggregates based on micromechanical model compared to the modulus $E_{a}^{\text {estimated }}$ estimated from the elastic properties of wood in its directions of anisotropy (literature data), for different values of Poisson's ratio.

\begin{tabular}{|l|c|c|c|}
\hline Poisson's ratio $\nu_{a}(-)$ & 0.1 & 0.2 & 0.3 \\
\hline$E_{a}^{\text {calibrated }}(\mathrm{MPa})$ & 1192 & 1237 & 1247 \\
$E_{a}^{\text {estimated }}(\mathrm{MPa})$ & 1232 & 924 & 616 \\
\hline
\end{tabular}

The second assumption to be discussed is the temporal constancy of the Young's modulus $E_{a}$ of the wood aggregates. This assumption is not always valid, especially in the presence of drying, since the mechanical properties of wood depend on its water content. If the water content of wood is modified from 25 to $12 \%$ (corresponding to a change of relative humidity from $100 \%$ to $60 \%$ ), its elastic properties will increase by 15 to around $25 \%$ [15]. For the spruce wood, in addition to the measurements presented in section 2.2 , we measured the Young's moduli at a water content of $25 \%$. The longitudinal Young's modulus decreased from $12760 \mathrm{MPa}$ (value estimated by using Eq. 
(2)) for a wood with a water content of $12 \%$ to $10300 \mathrm{MPa}$ for a wood with water content of $25 \%$. It may therefore be important to take into account this variation in the modeling. To quantify the effect of a change in the Young's modulus of wood aggregates on the predicted stiffness of the concrete, we compared the results obtained with the back-calculated value to those obtained with an increase and a decrease of this back-calculated value. Figure 11b illustrates this comparison for the concrete at 14 days. In this figure, we can see that modifying the Young's modulus of the aggregates by $\pm 25 \%$ induces a variation of the Young's moduli of the concrete ranging from $\pm 8 \%$ for a porosity of 0.1 to $\pm 17 \%$ for a porosity of 0.48 . The order of magnitude remains however correct.

The third assumption is the fact that we considered identical elastic properties of cement paste for the four studied mixtures of wood-aggregate concrete. This assumption seems to be strong, since the cement paste may present different microstructures due to the difference of $c / a$ ratios. Satisfactory results are, however, produced by the model using this assumption, indicating that it remains reasonable in the conditions of the present study. The amount of wood aggregates is probably so high that it influences in identical way the cement pastes in the range of this work.

In conclusion of this discussion, we can confirm the validity of the developed model. This model remains limited to the case of a concrete with an intergranular porosity of less than $50 \%$, which is the percolation limit of the self-consistent scheme. This threshold porosity, defined as the porosity 


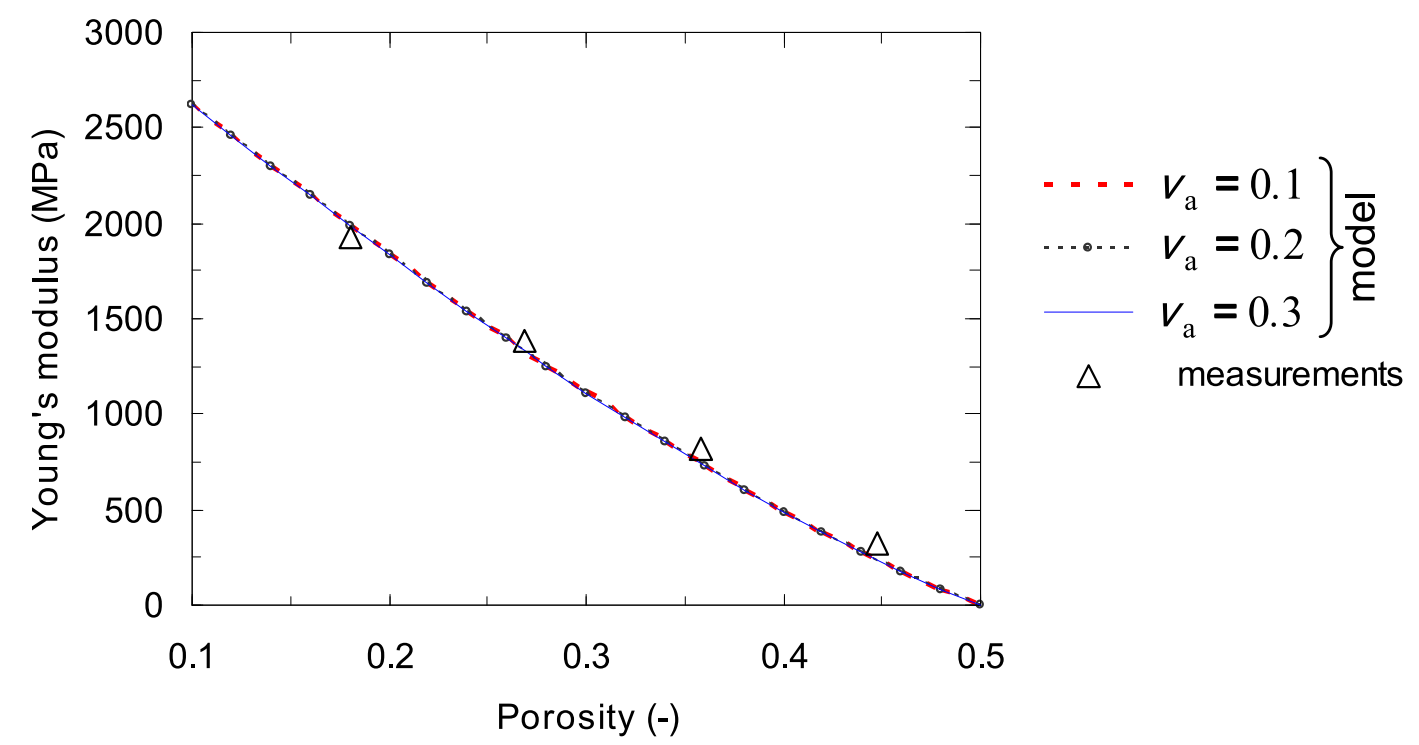

(a)

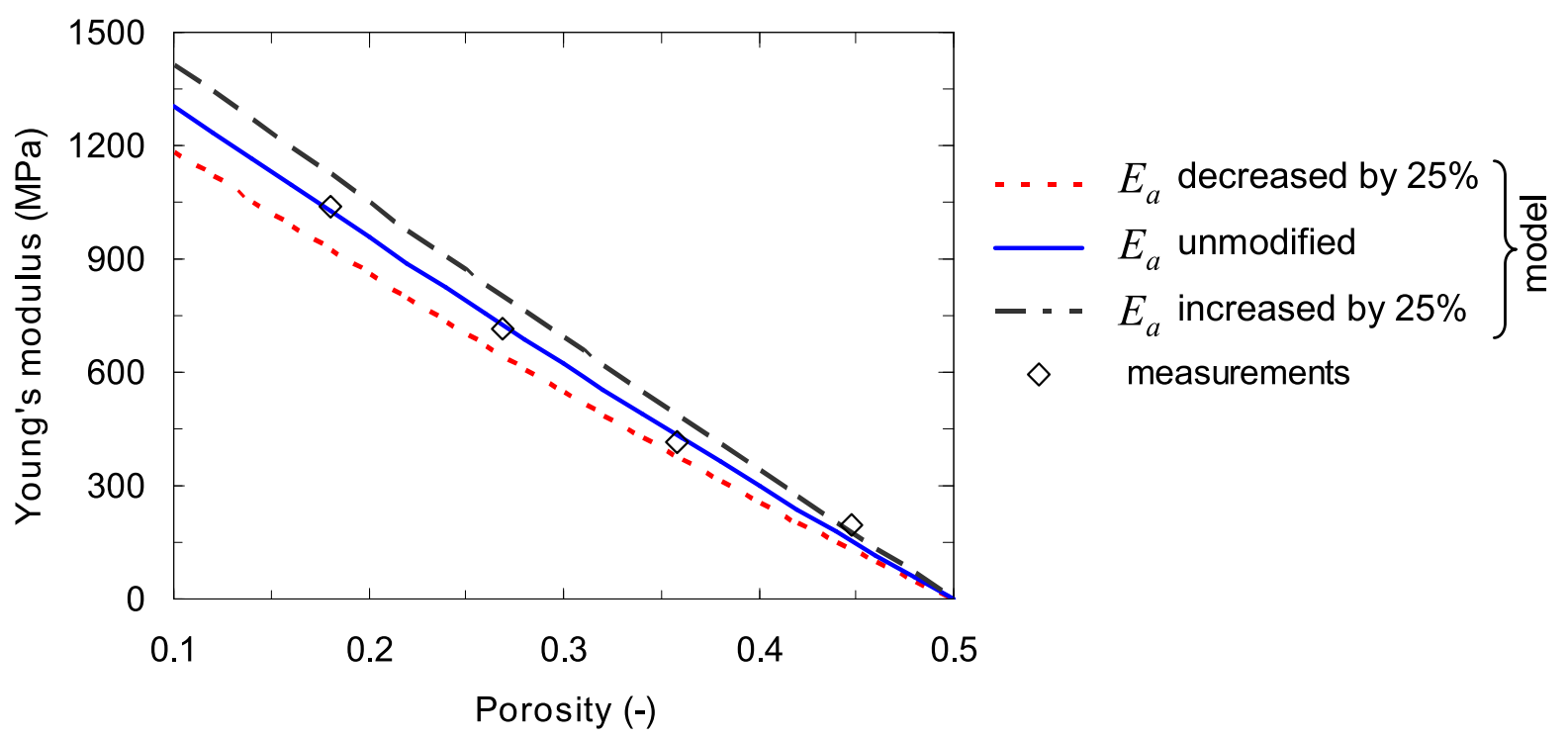

(b)

Figure 11: Effect of elastic properties of wood aggregates on the Young's moduli of woodaggregate concrete, as predicted with the derived model:: (a) effect of Poisson's ratio (at 28 days) and (b) effect of Young's modulus (at 14 days). 
above which the homogenized Young's modulus vanishes, is obtained in our case for a cement-to-wood mass ratio of $0.95-0.96$. For concretes presenting an intergranular porosity higher than $50 \%$, an adaptation of the model to the case of ellipsoidal inclusions could be a promising solution. Indeed, for the self-consistent scheme, the percolation threshold depends on the aspect ratio of the inclusions [30].

\section{Conclusion}

In this work, we studied experimentally and theoretically the elastic properties of wood-aggregate concrete, which is a very complex material that requires original characterization methods. This study showed the potential of digital image correlation technique to overcome some difficulties related to the characterization of the elastic behavior of this material. However, further work is required to deal with other difficulties in particular regarding how to assess some properties of each constituent (i.e., of the cement paste and of the aggregates) after the mixing of the concrete, in particular: water content, elastic properties, and so on.

This study showed also that micromechanical models yield predictive tools for the behavior of such heterogeneous materials. It is however necessary to take into account the interactions (i.e., exchange of water, modification of hydration and so on) between the various constituents. These interactions prevent the use of the properties measured on each individual component as input parameters of the models. This result highlights the 
importance of combining modeling and experiment to better understand the phenomena involved in complex materials such as wood-aggregate concrete.

The work reported here supports some results in the literature. The comparison between the Young's modulus measured on a free piece of cement paste and the one back-calculated from the fit of the micromechanical model confirms the effect of the presence of plant-based particles on the hydration of cement. Moreover, the model here developed captures well experimental data despite not taking into account the form and anisotropy of wood aggregates. Therefore, for a heterogeneous material containing elongated particles whose behavior is anisotropic, the use of spherical inclusions is sufficient to predict the macroscopic behavior, when these particles are randomly oriented.

The parametric study in this work showed that some input parameters (e.g., elastic properties of wood aggregates) of the micromechanical model have less influence on the homogenized properties than others (e.g., elastic properties of cement paste, volume fractions of phases). This finding might be used to determine priorities in terms of needs of characterization of the constituents of wood-aggregate concrete after mixing, but also for other concretes whose microstructure is similar to that of wood-aggregate concrete, like hemp concrete, concrete with flax shives and other bio-based materials.

\section{Acknowledgment}

The authors would like to thank Lafarge for their supply of cement and Agresta Technologies for their supply of wood aggregates. We also wish to 
thank M. Bornert for his appreciable help in digital image correlation, and N.

Lenoir for the X-ray tomography image. Special thanks to École des Mines de Douai, and in particular to Prof. D. Damidot and D. Betrancourt for the DRX analysis of cement.

\section{References}

[1] F. Pacheco-Torgal, S. Jalali, Cementitious building materials reinforced with vegetable fibres: A review, Construction and Building Materials 25 (2) (2011) 575-581.

[2] S. Caré, A. Lakehal, Hygro-mechanical behaviour of vegetable concrete materials: influence of the inter-particular porosity, in: 15th International Conference on Experimental Mechanics, ICEM15, Porto/Portugal, 22-27, 2012.

[3] K. A. Rim, A. Ledhem, O. Douzane, R. Dheilly, M. Queneudec, Influence of the proportion of wood on the thermal and mechanical performances of clay-cement-wood composites, Cement and Concrete Composites 21 (4) (1999) 269-276.

[4] M. Bederina, L. Marmoret, K. Mezreb, M. Khenfer, A. Bali, M. Quéneudec, Effect of the addition of wood shavings on thermal conductivity of sand concretes: Experimental study and modelling, Construction and Building Materials 21 (3) (2007) 662-668. 
[5] A. Bouguerra, A. Ledhem, F. de Barquin, R. M. Dheilly, M. Quéneudec, Effect of microstructure on the mechanical and thermal properties of lightweight concrete prepared from clay, cement, and wood aggregates, Cement and Concrete Research 28 (8) (1998) 1179-1190.

[6] V. Cérézo, Mechanical, thermal and acoustical properties of concrete containing vegetable particles : experimental approach and modeling, Ph.D. thesis, Institut National des Sciences Appliquées de Lyon, 2005.

[7] T. T. Nguyen, V. Picandet, P. Carre, T. Lecompte, S. Amziane, C. Baley, Effect of compaction on mechanical and thermal properties of hemp concrete, European Journal of Environmental and Civil Engineering $14(5)(2010) 545-560$.

[8] A. Bouguerra, Contribution to the study of a procedure for evaluating clayey wastes : Hygrothermal behavior of tested materials, Ph.D. thesis, Institut National des Sciences Appliquées de Lyon, 1997.

[9] P. Monreal, Study of the feasibility of beet pulp-based lignocellulosic concrete: Physico-chemical treatments and assessment of the influence on mechanical, hydric and thermal performances, Ph.D. thesis, Université de Picardie Jules Vernes, 2007.

[10] E. Mougel, A. L. Beraldo, A. Zoulalian, Controlled dimensional variations of a wood-cement composite, Holzforschung-International Journal 
of the Biology, Chemistry, Physics and Technology of Wood 49 (5) (1995) $471-477$.

[11] A. M. Cheumani Yona, Study of the microstructure of wood/cement composites by proton NMR relaxometry, Ph.D. thesis, Université Bordeaux 1 and Université de Yaoundé I, 2009.

[12] T. T. Nguyen, V. Picandet, S. Amziane, C. Baley, Influence of compactness and hemp hurd characteristics on the mechanical properties of lime and hemp concrete, European Journal of Environmental and Civil Engineering 13 (9) (2009) 1039-1050.

[13] T. H. Pham, F. Julien, V. Picandet, P. Pilvin, Étude expérimentale, théorique et numérique de l'élasticité de composites chaux-chanvre, 21ème Congrès Français de Mécanique, 26 au 30 août 2013, Bordeaux, France (FR) .

[14] S. Mom, S. Dartois, A. Ben Hamida, H. Dumontet, H. Boussa, Non linear micromechanical modeling of hemp concretes, in: 15th European Conference on Composite Materials, ECCM15, Venice/Italy, 1-8, 2012.

[15] C. C. Gerhards, Effect of moisture content and temperature on the mechanical properties of wood: an analysis of immediate effects, Wood and Fiber Science 14 (1) (1982) 4-36.

[16] M. Bornert, J. J. Orteu, S. Roux, Corrélation d'images, in: M. Grédiac, 
F. Hild (Eds.), Mesures de champs et identification en mécanique des solides, Lavoisier, 2011.

[17] M. Bornert, F. Vales, H. Gharbi, D. Nguyen Minh, Multiscale FullField Strain Measurements for Micromechanical Investigations of the Hydromechanical Behaviour of Clayey Rocks, Strain 46 (1) (2010) 3346.

[18] R. Le Roy, Instantaneous and deferred deformations of high-performance concrete, Ph.D. thesis, École Nationale des Ponts et Chaussées, 1995.

[19] D. Guitard, Mécanique du matériau bois et composites, Cépaduès, 1987.

[20] P. Navi, F. Heger, Comportement thermo-hydromécanique du bois, Presses polytechniques et universitaires romandes, 2005.

[21] Wood handbook: wood as an engineering material, General technical report FPL; GTR-113. Madison, WI: U.S. Department of Agriculture, Forest Service, Forest Products Laboratory: xi, [463] pages, 1999.

[22] A. Akkaoui, Wood aggregate concretes: experimental and theoretical study of thermo-hydro-mechanical properties using multi-scale approaches, Ph.D. thesis, Université Paris Est, 2014.

[23] L. Dormieux, D. Kondo, F. J. Ulm, Microporomechanics, John Wiley \& Sons, 2006. 
[24] R. M. Christensen, K. H. Lo, Solutions for effective shear properties in three phase sphere and cylinder models, Journal of the Mechanics and Physics of Solids 27 (4) (1979) 315 - 330.

[25] E. Hervé, A. Zaoui, n-Layered inclusion-based micromechanical modelling, International Journal of Engineering Science 31 (1) (1993) 1 10.

[26] S. Caré, E. Hervé, Application of a n-phase model to the diffusion coefficient of chloride in mortar, Transport in Porous Media 56 (2) (2004) $119-135$.

[27] E. Hervé, S. Caré, J. P. Seguin, Influence of the porosity gradient in cement paste matrix on the mechanical behavior of mortar, Cement and Concrete Research 40 (7) (2010) 1060-1071.

[28] A. Govin, A. Peschard, R. Guyonnet, Modification of cement hydration at early ages by natural and heated wood, Cement and Concrete Composites 28 (1) (2006) 12-20.

[29] R. J. Flatt, G. W. Scherer, J. W. Bullard, Why alite stops hydrating below $80 \%$ relative humidity, Cement and Concrete Research 41 (9) (2011) 987-992.

[30] J. Sanahuja, L. Dormieux, S. Meille, C. Hellmich, A. Fritsch, Micromechanical explanation of elasticity and strength of gypsum: from elon- 
681

682

683

684

gated anisotropic crystals to isotropic porous polycrystals, Journal of engineering mechanics 136 (2) (2009) 239-253.

[31] A. E. H. Love, A treatise on the mathematical theory of elasticity, Dover, New York, 1944. 


\section{Appendix A. Two-layered composite sphere problem in elasticity}

We consider a representative elementary volume of wood-aggregate con-

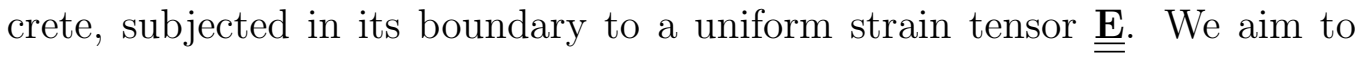
determine the microscopic strain tensor at each phase (cement paste and wood aggregates). To do so, we use the microstructural cell presented in Figure 8. This microstructural cell is embedded in an infinite elastic homogeneous medium having the stiffness tensor $\mathbb{C}_{h}$, and subjected to an auxiliary uniform strain tensor $\underline{\underline{\mathbf{E}}}^{\infty}$ at infinity.

To solve the problem easily, we first consider a spherical macroscopic strain $\underline{\mathbf{E}}^{\infty}=\mathbf{E}^{\infty} \underline{\underline{I}}$ at infinity. The displacement field solution of the problem in each phase $i(i=c, a, h)$ is looked for in the form (Love [31]):

$$
\boldsymbol{\xi}_{r}^{i}=\mathcal{X}_{i} r+\frac{\mathcal{Y}_{i}}{r^{2}}, \quad \boldsymbol{\xi}_{\theta}^{i}=\boldsymbol{\xi}_{\phi}^{i}=0
$$

where we used the spherical coordinates $(r, \theta, \phi)$, and $\mathcal{X}_{i}$ and $\mathcal{Y}_{i}$ are scalar constants to be determined. The stress tensor in each phase $i$ is given by:

$$
\boldsymbol{\sigma}_{r r}^{i}=3 k_{i} \mathcal{X}_{i}-4 \mu_{i} \frac{\mathcal{Y}_{i}}{r^{3}}, \quad \boldsymbol{\sigma}_{\theta \theta}^{i}=\boldsymbol{\sigma}_{\phi \phi}^{i}=3 k_{i} \mathcal{X}_{i}+2 \mu_{i} \frac{\mathcal{Y}_{i}}{r^{3}}, \quad \boldsymbol{\sigma}_{r \theta}=\boldsymbol{\sigma}_{r \phi}=\boldsymbol{\sigma}_{\theta \phi}=0
$$

where $k_{i}$ and $\mu_{i}$ are the bulk and the shear moduli of the phase $i$. The constant $\mathcal{X}_{a}$ vanishes to avoid a singularity at the center of the composite sphere; and from the boundary condition at infinity, we get: $\mathcal{X}_{h}=\mathbf{E}^{\infty}$. The other constants are determined by the condition of the continuity of stress 
and displacement fields at the interfaces $r=R_{a}$ and $r=R_{c}$.

We now examine the case of a purely deviatoric macroscopic strain $\underline{\mathbf{E}}^{\infty}=$ $\mathbf{E}^{\infty}\left(\underline{\mathbf{e}}_{1} \otimes \underline{\mathbf{e}}_{2}+\underline{\mathbf{e}}_{2} \otimes \underline{\mathbf{e}}_{1}\right)$ at infinity. The displacement field solution of the problem in each phase is looked for in the form (Love [31]):

$$
\left\{\begin{array}{l}
\boldsymbol{\xi}_{r}^{i}=\Gamma_{r}^{i}(r) \sin ^{2} \theta \cos 2 \phi \\
\boldsymbol{\xi}_{\theta}^{i}=\Gamma_{\theta}^{i}(r) \sin \theta \cos \theta \cos 2 \phi \\
\boldsymbol{\xi}_{\phi}^{i}=\Gamma_{\phi}^{i}(r) \sin \theta \sin 2 \phi
\end{array}\right.
$$

with $\Gamma_{r}^{i}, \Gamma_{\theta}^{i}$ and $\Gamma_{\phi}^{i}$ scalar functions that depend only on $r$, and whose expressions are given by the following equations:

$$
\left\{\begin{array}{l}
\Gamma_{r}^{i}=\mathcal{S}_{i} r-6 \frac{\nu_{i}}{1-2 \nu_{i}} \mathcal{T}_{i} r^{3}+3 \frac{\mathcal{U}_{i}}{r^{4}}+\frac{5-4 \nu_{i}}{1-2 \nu_{i}} \frac{\mathcal{V}_{i}}{r^{2}} \\
\Gamma_{\theta}^{i}=\mathcal{S}_{i} r-\frac{7-4 \nu_{i}}{1-2 \nu_{i}} \mathcal{T}_{i} r^{3}-2 \frac{\mathcal{U}_{i}}{r^{4}}+2 \frac{\mathcal{V}_{i}}{r^{2}} \\
\Gamma_{\phi}^{i}=-\Gamma_{\theta}^{i}
\end{array}\right.
$$

where: $\nu_{i}$ is the Poisson's ratio of the phase $i$; and $\mathcal{S}_{i}, \mathcal{T}_{i}, \mathcal{U}_{i}$ and $\mathcal{V}_{i}$ are scalar constants to be determined. The stress tensor resulting from the loading can be determined in each phase from its constitutive law as follows:

$$
\underline{\underline{\boldsymbol{\sigma}}}^{i}=\mathbb{C}_{i}: \nabla \underline{\boldsymbol{\xi}}^{i}
$$

Here we give the expressions of $\boldsymbol{\sigma}_{r r}$ and $\boldsymbol{\sigma}_{r \theta}$ that we need to determine the 
constants:

$$
\left\{\begin{array}{c}
\boldsymbol{\sigma}_{r r}^{i}=\left(2 \mu_{i} \mathcal{S}_{i}+\left(3 k_{i}-2 \mu_{i}\right) \mathcal{T}_{i} r^{2}-\frac{24 \mu_{i}}{r^{5}} \mathcal{U}_{i}-\frac{18 k_{i}+8 \mu_{i}}{r^{3}} \mathcal{V}_{i}\right) \sin ^{2} \theta \cos 2 \phi \\
\boldsymbol{\sigma}_{r \theta}^{i}=\left(2 \mu_{i} \mathcal{S}_{i}-\frac{2}{3}\left(24 k_{i}+5 \mu_{i}\right) \mathcal{T}_{i} r^{2}+\frac{16 \mu_{i}}{r^{5}} \mathcal{U}_{i}+\frac{6 k_{i}}{r^{3}} \mathcal{V}_{i}\right) \sin \theta \cos \theta \cos 2 \phi
\end{array}\right.
$$

where we replaced the Poisson's ratio by its expression as a function of the bulk and the shear moduli.

The constants $\mathcal{U}_{g}, \mathcal{V}_{g}$ and $\mathcal{T}_{h}$ vanish because of the boundary conditions at $r=0$ and $r \rightarrow \infty$; and from the boundary condition at infinity, we get: $\mathcal{S}_{h}=\mathbf{E}^{\infty}$. The other constants are determined by the condition of the continuity of stress $\left(\boldsymbol{\sigma}_{r r}\right.$ and $\left.\boldsymbol{\sigma}_{r \theta}\right)$ and displacement $\left(\boldsymbol{\xi}_{r}\right.$ and $\left.\boldsymbol{\xi}_{\theta}\right)$ fields at the interfaces $r=R_{a}$ and $r=R_{c}$.

\section{Appendix B. Determination of the bulk modulus of wood aggre- gates}

This section aims to determine the bulk modulus of wood aggregates basing on the Young's moduli and Poisson's ratios of wood in its directions of anisotropy. The bulk modulus $k$ of a material measures the material's resistance to uniform compression. It links the stress to the strain of the material. More precisely, $k$ is the proportionality coefficient between the isostatic stress $s$ and the isostatic strain rate $e$. We can then write:

$$
s=k e,
$$


with:

$$
s=\sigma_{11}=\sigma_{22}=\sigma_{33} \quad \text { and } \quad e=\varepsilon_{11}+\varepsilon_{22}+\varepsilon_{33} .
$$

${ }_{729}$ Wood is an orthotropic material. Its constitutive law can be written in 730 Voigt notation as follows:

$$
\left[\begin{array}{c}
\varepsilon_{l} \\
\varepsilon_{r} \\
\varepsilon_{t} \\
\gamma_{r t} \\
\gamma_{l t} \\
\gamma_{l r}
\end{array}\right]=\left[\begin{array}{cccccc}
\frac{1}{E_{l}} & \frac{-\nu_{r l}}{E_{r}} & \frac{-\nu_{t l}}{E_{t}} & 0 & 0 & 0 \\
\frac{-\nu_{l r}}{E_{l}} & \frac{1}{E_{r}} & \frac{-\nu_{t r}}{E_{t}} & 0 & 0 & 0 \\
\frac{-\nu_{l t}}{E_{l}} & \frac{-\nu_{r t}}{E_{r}} & \frac{1}{E_{t}} & 0 & 0 & 0 \\
0 & 0 & 0 & \frac{1}{\mu_{r t}} & 0 & 0 \\
0 & 0 & 0 & 0 & \frac{1}{\mu_{l t}} & 0 \\
0 & 0 & 0 & 0 & 0 & \frac{1}{\mu_{l r}}
\end{array}\right]\left[\begin{array}{c}
\sigma_{l} \\
\sigma_{r} \\
\sigma_{t} \\
\tau_{r t} \\
\tau_{l t} \\
\tau_{l r}
\end{array}\right],
$$

731 with:

$$
\frac{-\nu_{l r}}{E_{l}}=\frac{-\nu_{r l}}{E_{r}}, \quad \frac{-\nu_{l t}}{E_{l}}=\frac{-\nu_{t l}}{E_{t}}, \quad \frac{-\nu_{r t}}{E_{r}}=\frac{-\nu_{t r}}{E_{t}} .
$$

${ }_{732}$ Using this constitutive law, we can express $e^{a}=\varepsilon_{l}+\varepsilon_{r}+\varepsilon_{r}$ as a function 733 of $s^{a}=\frac{1}{3}\left(\sigma_{l}+\sigma_{r}+\sigma_{t}\right)$ taking into account the fact that $\sigma_{l}=\sigma_{r}=\sigma_{t}$. And 734 using this expression together with equation (B.1), the following equation 735 yields:

$$
\frac{1}{k^{a}}=\frac{1-\nu_{l r}-\nu_{l t}}{E_{l}}+\frac{1-\nu_{r l}-\nu_{r t}}{E_{r}}+\frac{1-\nu_{t r}-\nu_{t l}}{E_{t}} .
$$

